\title{
Dynkin diagrams and spectra of graphs
}

\author{
Michael A. Dokuchaev
}

Departamento de Matematica Univ. de São Paulo, Caixa Postal 66281, São Paulo, SP 05315-970 - Brazil

\section{Nadiya M. Gubareni}

Institute of Econometry \& Computer Science, Department of Management Technical Univ. of Czestochowa, Dabrowskiego str., 69, 42-200 Czestochowa, Poland

\section{Vyacheslav M. Futorny}

Departamento de Matematica Univ. de São Paulo, Caixa Postal 66281, São Paulo, SP 05315-970 - Brazil

\section{Marina A. Khibina}

In-t of Eng. Thermophysics of NAS of Ukraine, Zheljabova Str., 2A, 03057, Kiev, Ukraine

\section{Vladimir V. Kirichenko}

Faculty of Mechanics and Mathematics, Kiev National, Taras Shevchenko Univ., Vladimirskaya Str., 64, 01033 Kiev, Ukraine

\section{Introduction}

Dynkin diagrams first appeared in [20] in the connection with classification of simple Lie groups. Among Dynkin diagrams a special role is played by the simply laced Dynkin diagrams $A_{n}, D_{n}, E_{6}, E_{7}$ and $E_{8}$. Dynkin diagrams are closely related to Coxeter graphs that appeared in geometry (see [8]). After that Dynkin diagrams appeared in many braches of mathematics and beyond, em particular em representation theory.

In [22] P. Gabriel introduced a notion of a quiver (directed graph) and its representations. He proved the famous Gabriel's theorem on representations of quivers over algebraically closed field.

Let $Q$ be a finite quiver and $\bar{Q}$ the undirected graph obtained from $Q$ by deleting the orientation of all arrows. 
Theorem 1.1. (Gabriel's Theorem). A connected quiver $Q$ is of finite type if and only if the graph $\bar{Q}$ is one of the following simply laced Dynkin diagrams: $A_{n}, D_{n}, E_{6}, E_{7}$ or $E_{8}$.

I.N. Bernstein, I.M. Gelfand and V.A. Ponomarev [5] gave a proof of Gabriel's Theorem using roots, Weyl groups and Coxeter functors.

The terms "tame type" and "wild type" were introduced by P. Donovan and M.R. Freislich [16]. Extended Dynkin diagrams or Euclidean diagrams are $\tilde{A}_{n}, \tilde{D}_{n}, \tilde{E}_{6}, \tilde{E}_{7}$ and $\tilde{E}_{8}$ (see, for example, [2]). Tame quivers in terms of extended Dynkin diagrams were classified by L.A. Nazarova [39] and by P. Donovan-M.R. Freislich [16]. For finite dimensional algebras and some other algebraic structures the tame-wild dichotomy problem was solved by Yu.A. Drozd [17]-[19]. The theory of $K$-species was first considered by P. Gabriel in [23]. He obtained the characterization of $K$-species of finite type in a special case. His result was extended by V. Dlab and C.M. Ringel (see [14, Theorem B]).

Theorem 1.2. (Theorem B). A K-species is of a finite type if and only if its diagram is a finite disjoint union of Dynkin diagrams.

The problem of the ubiquity of the symply laced Dynkin diagrams $A_{n}$, $D_{n}, E_{n}$ was formulated by V.I. Arnold [1] as follows.

A-D-E classification. The Coxeter-Dynkin graphs $A_{n}, D_{n}$ and $E_{n}$ appear in many independent classification theorems. For instance

(a) the classification of the platonic solids (or finite orthogonal groups in euclidean 3-space),

(b) the classification of the categories of linear spaces and maps (representations of quivers,

(c) the classification of the singularities of algebraic hypersurfaces, with a definite intersection form of the neighboring smooth fibre,

(d) the classification of the critical points of functions having no moduli,

(e) the classification of the Coxeter groups generated by reflections, or, of Weyl groups with roots of equal length.

The problem is to find the common origin of all A-D-E classification theorems and to substitute a priori proofs to a posteriori verifications of the parallelism of the classifications. An introduction to the A-D-E-problem can be found in [30].

Dynkin diagrams and extended Dynkin diagrams are widely used in the study of generalized Cartan matrices and Kac-Moody algebras [2]-[4], [6], [31], [35], [36], [40] and [42]. 
Let $G$ be a finite graph without loops and multiple edges ( $G$ is a finite simple graph). J.H. Smith [41] formulated the following result:

Theorem 1.3. Let $G$ be a finite simple graph with the spectral radius (index) $r_{G}$. Then $r_{G}=2$ if and only if each connected component of $G$ is one of the extended Dynkin diagram $\tilde{A}_{n}, \tilde{D}_{n}, \tilde{E}_{6}, \tilde{E}_{7}, \tilde{E}_{8}$. Moreover, $r_{G}<2$ if and only if each connected component of $G$ is one of Dynkin diagrams $A_{n}$, $D_{n}, E_{6}, E_{7}, E_{8}$.

For the full proofs of this Smith's theorem see, for example, [27, chapter I and Appendix I], [37] and [21, Theorem 2.12]. Note that Theorem 1.3 was obtained also in [33, Theorem 5.1] and [7]. In 1975 (see [11]) D.M. Cvetkovich and I. Gutman introduced for extended Dynkin diagrams of type $\tilde{A}$ and $\tilde{D}$ the symbols $C_{n}$ and $W_{n}$. Moreover, they used the following notations: $P_{n}$ for $A_{n} ; Z_{n}$ for $D_{n+2}, T_{1}$ for $E_{6}, T_{2}$ for $E_{7}, T_{3}$ for $E_{8}, T_{4}$ for $\tilde{E}_{6}, T_{5}$ for $\tilde{E}_{7}$ and $T_{6}$ for $\tilde{E}_{8}$.

The following terminology is used in [12, pp. 77-79]: "Smith's graphs" means extended Dynkin diagrams and "reduced Smith's graphs" means simply laced Dynkin diagrams $A_{n}, D_{n}, E_{6}, E_{7}, E_{8}$ (see also [9] and [10]).

In this paper we consider spectral properties of graphs based on PerronFrobenius theory of non-negative matrices. We will use terminology and results from [29, Section 6.5] and [25].

\section{Symmetric non-negative matrices}

Let $G$ be an undirected finite graph without loops and multiple edges, i.e., $G$ is a finite simple graph.

Let $V G=\{1, \ldots, n\}$ be the vertex set of $G$ and $E G$ be the edge set of $\mathrm{G}$. Two vertices $i$ and $j$ are called adjacent if they are connected by an edge.

The adjacency matrix $[G]$ of a simple graph with $n$ vertices is a square matrix $[G]=\left(\alpha_{j}\right)$ of order $n$, whose $(i, j)$-entry $\alpha_{i j}$ is 1 , if the vertices $i$ and $j$ are adjacent, otherwise $\alpha_{i j}=0$. Therefore, $[G]$ is a symmetric $(0,1)$-matrix with zero main diagonal.

Denote by $M_{n}(\mathbb{R})$ the ring of all $n \times n$ matrices with real entries. Let $A=$ $\left(a_{i j}\right) \in M_{n}(\mathbb{R})$ be a non-negative symmetric permutationally irreducible matrix.

From the Perron-Frobenius Theorem it follows that $A$ has the largest positive eigenvalue $r_{A}$ such that any eigenvalue $\lambda$ of $A$ one has that $|\lambda| \leq r_{A}$, and there exists a positive eigenvector $\vec{z}=\left(z_{1}, \ldots, z_{n}\right)^{T}$ with $A \vec{z}=r_{A} \vec{z}$. We give the next. 
Theorem 2.1. Let $A=\left(a_{i j}\right) \in M_{n}(\mathbb{R})$ be a nonnegative symmetric permutationally irreducible matrix and $B$ be its proper main submatrix. Then $r_{B}<r_{A}$.

Before the proof of the theorem we give necessary information about the properties of $A$.

Lemma 2.1. [26] Eigenvectors of a matrix belonging to different eigenvalues are orthogonal.

Corollary 2.1. Let $A \in M_{n}(\mathbb{R})$ be a permutationally irreducible symmetric matrix and $\vec{z}=\left(z_{1}, \ldots, z_{n}\right)^{T}$ be its positive eigenvector, then $A \vec{z}=r_{A} \vec{z}$.

Proof. Suppose that $A \vec{z}=\lambda \vec{z}$ and $\lambda \neq r_{A}$. Let $\vec{w}=\left(w_{1}, \ldots, w_{n}\right)^{T}$ be a positive eigenvector of $A$ with eigenvalue $r_{A}$. Then by Lemma 2.1 the inner product $(\vec{z}, \vec{w})$ is zero. We obtain a contradiction:

$$
\sum_{i=1}^{n} z_{i} w_{i}>0
$$

Now we give a proof of Theorem 2.1.

Proof. Let $B$ be a proper principal $m \times m$-submatrix of $A$. We enumerate the rows and columns of $A$ such that:

$$
A=\left(\begin{array}{ccc|c}
B_{1} & \ldots & 0 & X_{1} \\
\vdots & \ddots & \vdots & \vdots \\
0 & \ldots & B_{t} & X_{t} \\
\hline X_{1}^{T} & \ldots & X_{t}^{T} & \mathrm{C}
\end{array}\right)
$$

where $B=\left(\begin{array}{ccc}B_{1} & \ldots & 0 \\ \vdots & \ddots & \vdots \\ 0 & \ldots & B_{t}\end{array}\right)$ and the matrices $B_{1}, \ldots, B_{t}$ are permutationally irreducible.

We may assume that $r_{B}=r_{B_{1}}, B_{1} \in M_{m_{1}}(\mathbb{R}), \ldots, B_{t} \in M_{m_{t}}(\mathbb{R})$, $m_{1}+\ldots+m_{t}=m$. Then, $C \in M_{n-m}(\mathbb{R})$ and $X=\left(\begin{array}{c}X_{1} \\ \vdots \\ X_{t}\end{array}\right)$, where $X_{i} \in M_{m_{i} \times(n-m)}(\mathbb{R})$.

The matrix $A$ is permutationally irreducible, so $X_{1} \neq 0$. 
Let $\vec{z}=\left(z_{1}, \ldots, z_{n}\right)^{T}$ be the Perron-Frobenius positive eigenvector of $A$, i.e., $A \vec{z}=r_{A} \vec{z}$. Denote by $\vec{z}_{s}=\left(z_{1}, \ldots, z_{m_{1}}\right)$ th evector formed by the first $m_{1}$ coordinates of $\vec{z}$ and by $\vec{z}_{e}=\left(z_{n-m+1}, \ldots, z_{n}\right)$.

Then we obtain: $B_{1} \vec{z}_{s}+X_{1} \vec{z}_{e}=r_{A} \vec{z}_{s}$. Obviously the non-negative vector $X_{1} \vec{z}_{e}$ is nonzero (vector $\vec{z}_{e}$ is positive and $X_{1} \neq 0$ and non-negative). We have $y_{i} \geq 0$ for $i=1, \ldots, m_{1}$. Therefore $y_{i} \leq r_{A} z_{i}$ for $i=1, \ldots, m_{1}$ and there exists $1 \leq k \leq m_{1}$ such that $y_{k}<r_{A} z_{k}$. Let $\vec{f}=\left(f_{1}, \ldots, f_{m_{1}}\right)^{T}$ be a Perron-Frobenius vector of $B_{1}$, so $B_{1} \vec{f}=r_{B} \vec{f}$. Then $\left(\vec{z}_{s}, B_{1} \vec{f}\right)=$ $\left(\vec{z}_{s}, r_{B_{1}} \vec{f}\right)=r_{B_{1}}\left(\vec{z}_{s}, \vec{f}\right)=\left(B_{1} \vec{z}_{s}, \vec{f}\right)<\left(r_{A} \vec{z}_{s}, \vec{f}\right)=r_{A}\left(\vec{z}_{s}, \vec{f}\right)$, i.e., $r_{B_{1}}\left(\vec{z}_{s}, \vec{f}\right)<$ $r_{A}\left(\vec{z}_{s}, \vec{f}\right)$. Then $\left.\vec{z}_{s}, \vec{f}\right)>0$ as inner product of positive vectors. Therefore $r_{B}=r_{B_{1}}<r_{A}$. Theorem is proved.

\section{Spectra of Dynkin diagrams and extended Dynkin dia- grams}

In this section we give a list of characteristic polynomials and spectra of Dynkin diagrams and of extended Dynkin diagrams.

Theorem 3.1. (L. Kronecker, [32]) Suppose that all the real roots of a monic polynomial with integer coefficients belong to the interval $[-2,2]$ and are given in the form

$$
2 \cos \alpha, 2 \cos \beta, 2 \cos \gamma, \ldots
$$

Then the angles $\alpha, \beta, \gamma, \ldots$ are rational multiples of $\pi / 2$.

The following simple graphs are simply laced Dynkin diagrams:

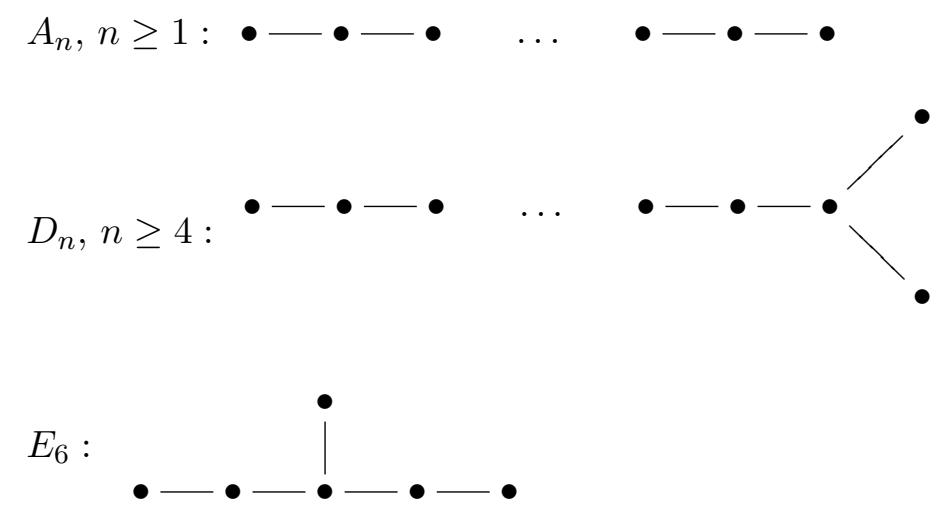




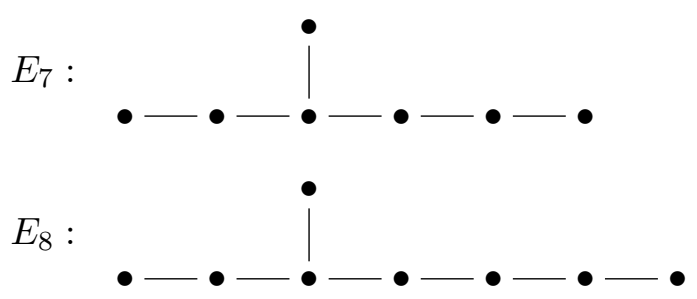

The following simple graphs are extended versions of simply laced Dynkin diagrams:

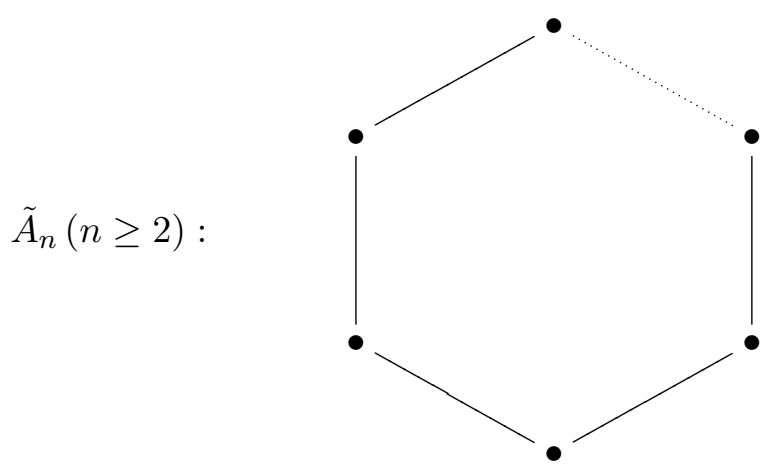

$\tilde{D}_{n}(n \geq 4):$
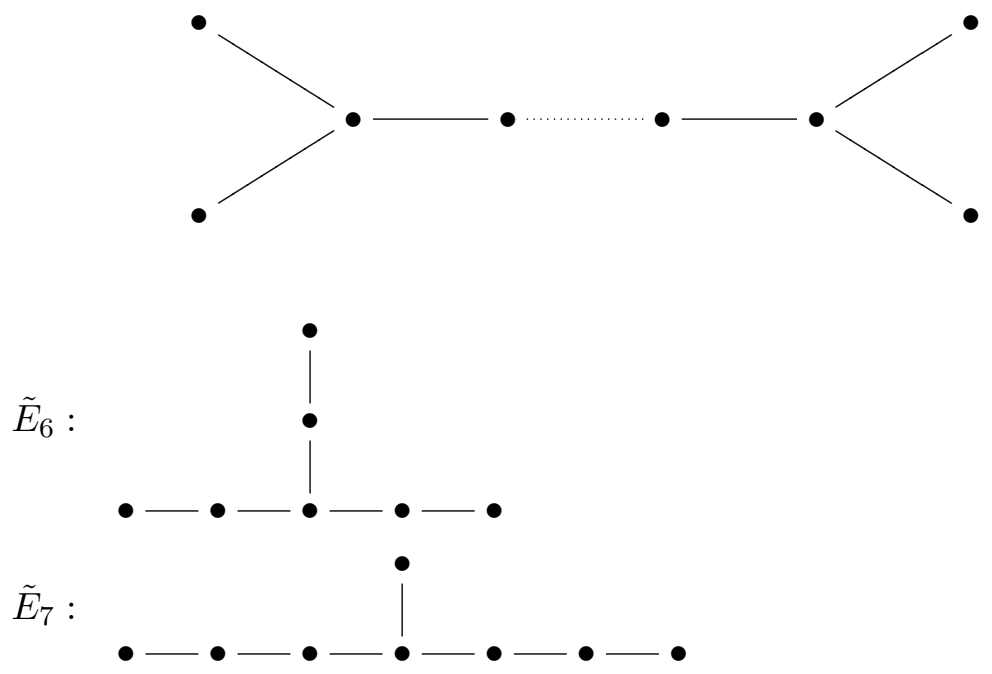

São Paulo J.Math.Sci. 7, 1 (2013), 83-104 


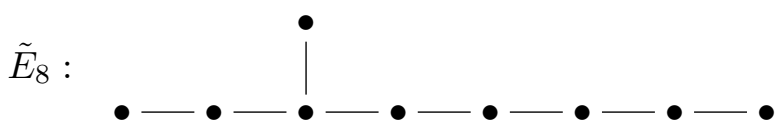

Often extended Dynkin diagrams are called Euclidean diagrams.

Proposition 3.1. For the Dynkin diagram $A_{n}(n \geq 1)$ we have

$$
\chi_{A_{n}}(x)=\prod_{1 \leq k \leq n}\left(x-2 \cos \frac{k \pi}{n+1}\right)
$$

Consequently,

$$
S\left(A_{n}\right)=\left\{2 \cos \frac{k \pi}{n+1} \mid k=1, \ldots, n\right\}
$$

and $r_{A_{n}}=2 \cos \frac{\pi}{n+1}$, where $S\left(A_{n}\right)$ denotes the spectrum of $A_{n}$.

Proposition 3.2. For the Dynkin diagram $D_{n}(n \geq 4)$ we have

$$
\chi_{D_{n}}(x)=x\left(\prod_{0 \leq k \leq n-2}\left(x-2 \cos \frac{(1+2 k) \pi}{2(k-1)}\right) .\right.
$$

Consequently, $S\left(D_{n}\right)$ consists of zero and of the following set:

$$
\left\{2 \cos \frac{(1+2 k) \pi}{2(n-1)} \mid k=0, \ldots, n-2\right\}
$$

and $r_{D_{n}}=2 \cos \frac{\pi}{2(n-1)}$.

Proposition 3.3. For the Dynkin diagram $E_{6}$ we have

$$
\chi_{E_{6}}(x)=x^{6}-5 x^{4}+5 x^{2}-1=\prod_{1 \leq k \leq 6}\left(x-2 \cos \frac{m_{k} \pi}{12}\right),
$$

where $m_{k}=1,4,5,7,8,11$. Then

and $r_{E_{6}}=2 \cos \frac{\pi}{12}$.

$$
S\left(E_{6}\right)=\left\{2 \cos \frac{m_{k} \pi}{12} \mid m_{k}=1,4,5,7,8,11\right\}
$$

Proposition 3.4. For the Dynkin diagram $E_{7}$ we have

$$
\chi_{E_{7}}(x)=x\left(x^{6}-6 x^{4}+9 x^{2}-3\right)=\prod_{1 \leq k \leq 7}\left(x-2 \cos \frac{m_{k} \pi}{18}\right),
$$


where $m_{k}=1,5,7,9,11,13,17$. Then

$$
S\left(E_{7}\right)=\left\{2 \cos \frac{m_{k} \pi}{18} \mid m_{k}=1,5,7,9,11,13,17\right\}
$$

and $r_{E_{7}}=2 \cos \frac{\pi}{18}$.

Proposition 3.5. For the Dynkin diagram $E_{8}$ we have

$$
\chi_{E_{8}}(x)=x^{8}-7 x^{6}+14 x^{4}-8 x^{2}+1=\prod_{1 \leq k \leq 8}\left(x-2 \cos \frac{m_{k} \pi}{30}\right),
$$

where $m_{k}=1,7,11,13,17,19,23,29$. Then

$$
S\left(E_{8}\right)=\left\{2 \cos \frac{m_{k} \pi}{30} \mid m_{k}=1,7,11,13,17,19,23,29\right\}
$$

and $r_{E_{8}}=2 \cos \frac{\pi}{30}$.

Proposition 3.6. For the extended Dynkin diagram $\tilde{A}_{n}(n \geq 2)$ we have

$$
\chi_{\tilde{A}_{n}}(x)=\mu^{n+1}+\mu^{-n-1}-2=\prod_{1 \leq k \leq n}\left(x-2 \cos \frac{2 k \pi}{n+1}\right),
$$

where $x=\mu+\frac{1}{\mu}$. Then consequently,

$$
S\left(\tilde{A}_{n}\right)=\left\{2 \cos \frac{2 k \pi}{n+1} \mid k=0, \ldots, n\right\}
$$

and $r_{\tilde{A}_{n}}=2$.

Proposition 3.7. For the extended Dynkin diagram $\tilde{D}_{n}(n \geq 4)$ we have

$$
\chi_{\tilde{D}_{n}}(x)=\chi_{\tilde{A}_{3}}(x) \chi_{n-3}(x)=x^{2}\left(x^{2}-4\right) \prod_{0 \leq k \leq n-3}\left(x-2 \cos \frac{k \pi}{n-2}\right) .
$$

Then

$$
S\left(\tilde{D}_{n}\right)=\left\{2 \cos \frac{k \pi}{n-2} \mid k=1, \ldots, n-3\right\} \cup[-2,0,0,2]
$$

and $r_{\tilde{D}_{n}}=2$. 
Proposition 3.8. For the extended Dynkin diagrams $\tilde{E}_{6}, \tilde{E}_{7}, \tilde{E}_{8}$ we have

$$
\begin{aligned}
& \chi_{\tilde{E}_{6}}(x)=x\left(x^{2}-1\right)^{2}\left(x^{2}-4\right) ; \\
& \chi_{\tilde{E}_{7}}(x)=x\left(x^{2}-1\right)\left(x^{2}-4\right) \prod_{1 \leq k \leq 3}\left(x-2 \cos \frac{k \pi}{4}\right) ; \\
& \chi_{\tilde{E}_{8}}(x)=x\left(x^{2}-1\right)\left(x^{2}-4\right) \prod_{1 \leq k \leq 4}\left(x-2 \cos \frac{k \pi}{5}\right) .
\end{aligned}
$$

Then

$$
\begin{aligned}
& S\left(\tilde{E}_{6}\right)=[0, \pm 1, \pm 1, \pm 2] \text { and } r_{\tilde{E}_{6}}=2 . \\
& S\left(\tilde{E}_{7}\right)=\left\{2 \cos \frac{k \pi}{4} \mid k=1,2,3\right\} \cup\{0, \pm 1, \pm 2\} \text { and } r_{\tilde{E}_{7}}=2 . \\
& S\left(\tilde{E}_{8}\right)=\left\{2 \cos \frac{k \pi}{5} \mid k=1,2,3,4\right\} \cup\{0, \pm 1, \pm 2\} \text { and } r_{\tilde{E}_{8}}=2 .
\end{aligned}
$$

\section{Perron-Frobenius vectors of extended Dynkin diagrams}

We consider simply laced extended Dynkin diagrams and its PerronFrobenius vectors.

We give the list of these graphs with the numbering of vertices suitable for us:

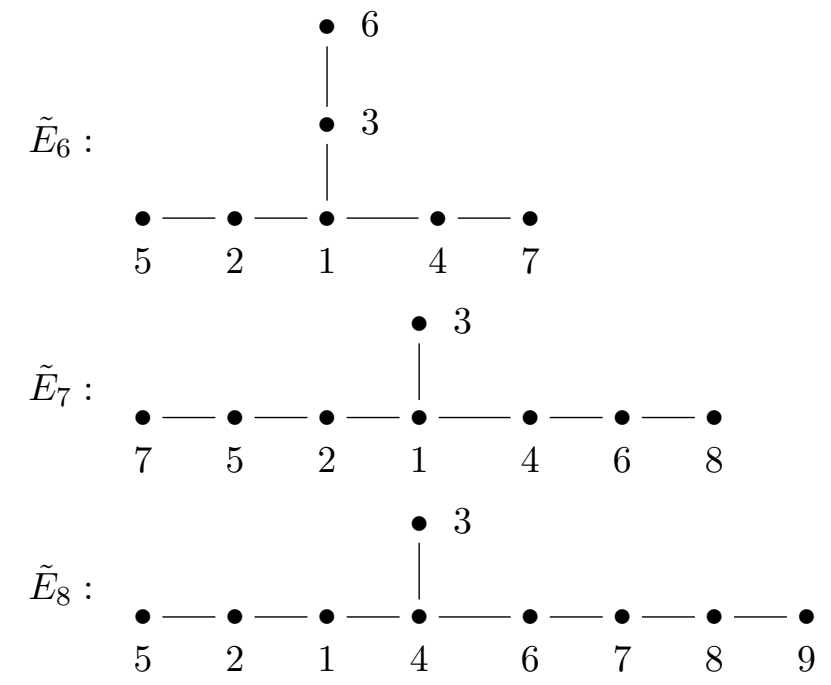




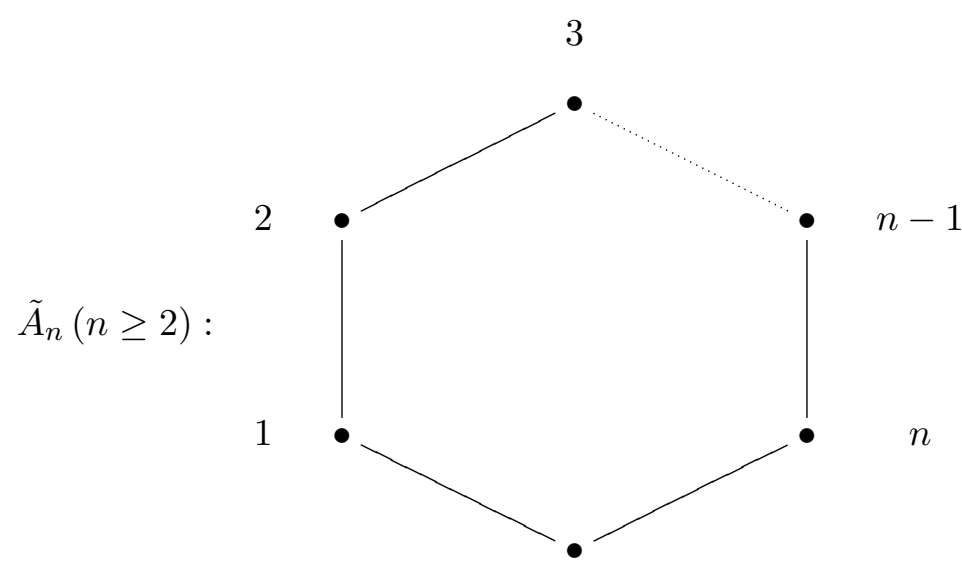

$n+1$

$\tilde{D}_{n}(n \geq 4)$ :

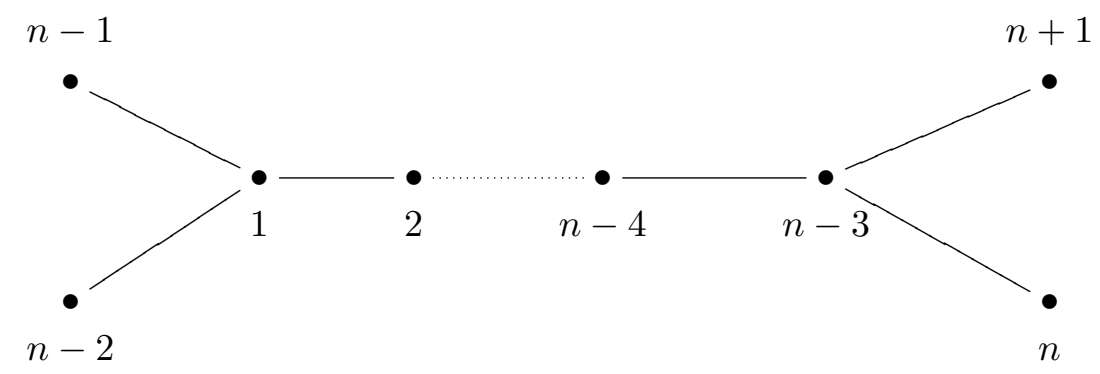

Case $\tilde{E}_{6}$.

The adjacency matrix is

$$
\left[\tilde{E}_{6}\right]=\left[\begin{array}{lllllll}
0 & 1 & 1 & 1 & 0 & 0 & 0 \\
1 & 0 & 0 & 0 & 1 & 0 & 0 \\
1 & 0 & 0 & 0 & 0 & 1 & 0 \\
1 & 0 & 0 & 0 & 0 & 0 & 1 \\
0 & 1 & 0 & 0 & 0 & 0 & 0 \\
0 & 0 & 1 & 0 & 0 & 0 & 0 \\
0 & 0 & 0 & 1 & 0 & 0 & 0
\end{array}\right]
$$

Let $\vec{z}=\left(z_{1}, z_{2}, z_{3}, z_{4}, z_{5}, z_{6}, z_{7}\right)^{T}$ be a positive eigenvector of $\tilde{E}_{6}$. 


$$
\begin{aligned}
& {\left[\begin{array}{lllllll}
0 & 1 & 1 & 1 & 0 & 0 & 0 \\
1 & 0 & 0 & 0 & 1 & 0 & 0 \\
1 & 0 & 0 & 0 & 0 & 1 & 0 \\
1 & 0 & 0 & 0 & 0 & 0 & 1 \\
0 & 1 & 0 & 0 & 0 & 0 & 0 \\
0 & 0 & 1 & 0 & 0 & 0 & 0 \\
0 & 0 & 0 & 1 & 0 & 0 & 0
\end{array}\right]\left[\begin{array}{l}
z_{1} \\
z_{2} \\
z_{3} \\
z_{4} \\
z_{5} \\
z_{6} \\
z_{7}
\end{array}\right]=\left[\begin{array}{l}
\lambda z_{1} \\
\lambda z_{2} \\
\lambda z_{3} \\
\lambda z_{4} \\
\lambda z_{5} \\
\lambda z_{6} \\
\lambda z_{7}
\end{array}\right]} \\
& \left\{\begin{aligned}
z_{2}+z_{3}+z_{4} & =\lambda z_{1} \\
z_{1}+z_{5} & =\lambda z_{2} \\
z_{1}+z_{6} & =\lambda z_{3} \\
z_{1}+z_{7} & =\lambda z_{4} \\
& z_{2}=\lambda z_{5} \\
z_{3} & =\lambda z_{6} \\
z_{4} & =\lambda z_{7}
\end{aligned}\right. \\
& z_{2}+z_{3}+z_{4}=\lambda\left(z_{5}+z_{6}+z_{7}\right)=\lambda z_{1} \\
& z_{1}=z_{5}+z_{6}+z_{7}, 4 z_{1}=\lambda^{2} z_{1} \text {, i.e., } \lambda=2 \text {. } \\
& z_{2}=2 z_{5}, z_{3}=2 z_{6} \text { and } z_{2}=z_{3}=z_{4}=2,2 z_{1}=6, z_{1}=3 \text {. }
\end{aligned}
$$

We obtain $\vec{z}=(3,2,2,2,1,1,1)^{T}$.

Case $\tilde{E}_{7}$.

The adjacency matrix is

$$
\left[\tilde{E}_{7}\right]=\left[\begin{array}{cccccccc}
0 & 1 & 1 & 1 & 0 & 0 & 0 & 0 \\
1 & 0 & 0 & 0 & 1 & 0 & 0 & 0 \\
1 & 0 & 0 & 0 & 0 & 0 & 0 & 0 \\
1 & 0 & 0 & 0 & 0 & 1 & 0 & 0 \\
0 & 1 & 0 & 0 & 0 & 0 & 1 & 0 \\
0 & 0 & 0 & 1 & 0 & 0 & 0 & 1 \\
0 & 0 & 0 & 0 & 1 & 0 & 0 & 0 \\
0 & 0 & 0 & 0 & 0 & 1 & 0 & 0
\end{array}\right]
$$

As above

$$
\left[\begin{array}{llllllll}
0 & 1 & 1 & 1 & 0 & 0 & 0 & 0 \\
1 & 0 & 0 & 0 & 1 & 0 & 0 & 0 \\
1 & 0 & 0 & 0 & 0 & 0 & 0 & 0 \\
1 & 0 & 0 & 0 & 0 & 1 & 0 & 0 \\
0 & 1 & 0 & 0 & 0 & 0 & 1 & 0 \\
0 & 0 & 0 & 1 & 0 & 0 & 0 & 1 \\
0 & 0 & 0 & 0 & 1 & 0 & 0 & 0 \\
0 & 0 & 0 & 0 & 0 & 1 & 0 & 0
\end{array}\right]\left[\begin{array}{c}
z_{1} \\
z_{2} \\
z_{3} \\
z_{4} \\
z_{5} \\
z_{6} \\
z_{7} \\
z_{8}
\end{array}\right]=\lambda\left[\begin{array}{c}
z_{1} \\
z_{2} \\
z_{3} \\
z_{4} \\
z_{5} \\
z_{6} \\
z_{7} \\
z_{8}
\end{array}\right]
$$




$$
\left\{\begin{aligned}
z_{1}+z_{3}+z_{4} & =\lambda z_{1} \\
z_{1}+z_{5} & =\lambda z_{2} \\
z_{1}+z_{6} & =\lambda z_{3} \\
z_{2}+z_{7} & =\lambda z_{5} \\
z_{4}+z_{8} & =\lambda z_{6} \\
z_{5} & =\lambda z_{7} \\
z_{6} & =\lambda z_{8}
\end{aligned}\right.
$$

We have $z_{6}=\lambda z_{8}, z_{4}=\left(\lambda^{2}-1\right) z_{8}, z_{1}=\left(\lambda^{3}-2 \lambda\right) z_{8}$,

$z_{3}=\left(\lambda^{2}-2\right) z_{8}, z_{2}=\left(\lambda^{4}-4 \lambda^{2}+3\right) z_{8}$,

$z_{5}=\left(\lambda^{5}-5 \lambda^{3}+5 \lambda\right) z_{8}, z_{7}=\left(\lambda^{4}-5 \lambda^{2}+5\right) z_{8}$.

Let $z_{8}=1$. Then from $z_{2}+z_{7}=\lambda z_{5}$ it follows that $\lambda^{4}-4 \lambda^{2}+3+\lambda^{4}-5 \lambda^{2}+5=\lambda^{6}-5 \lambda^{4}+5 \lambda^{2}$, i.e., $\lambda^{6}-7 \lambda^{4}+14 \lambda^{2}-8=0$.

Obviously, $2^{6}-7 \cdot 2^{4}+14 \cdot 2^{2}-2^{3}=2^{2}(16-28+14-2)=0$, i.e., $\lambda=2$ is a root. Therefore $\vec{z}=(4,3,2,3,2,2,1,1)^{T}$.

Case $\tilde{E}_{8}$.

The adjacency matrix is

$$
\left[\tilde{E}_{8}\right]=\left[\begin{array}{ccccccccc}
0 & 1 & 1 & 1 & 0 & 0 & 0 & 0 & 0 \\
1 & 0 & 0 & 0 & 1 & 0 & 0 & 0 & 0 \\
1 & 0 & 0 & 0 & 0 & 0 & 0 & 0 & 0 \\
1 & 0 & 0 & 0 & 0 & 1 & 0 & 0 & 0 \\
0 & 1 & 0 & 0 & 0 & 0 & 0 & 0 & 0 \\
0 & 0 & 0 & 1 & 0 & 0 & 1 & 0 & 0 \\
0 & 0 & 0 & 0 & 0 & 1 & 0 & 1 & 0 \\
0 & 0 & 0 & 0 & 0 & 0 & 1 & 0 & 1 \\
0 & 0 & 0 & 0 & 0 & 0 & 0 & 1 & 0
\end{array}\right]
$$

As above

$$
\left[\begin{array}{lllllllll}
0 & 1 & 1 & 1 & 0 & 0 & 0 & 0 & 0 \\
1 & 0 & 0 & 0 & 1 & 0 & 0 & 0 & 0 \\
1 & 0 & 0 & 0 & 0 & 0 & 0 & 0 & 0 \\
1 & 0 & 0 & 0 & 0 & 1 & 0 & 0 & 0 \\
0 & 1 & 0 & 0 & 0 & 0 & 0 & 0 & 0 \\
0 & 0 & 0 & 1 & 0 & 0 & 1 & 0 & 0 \\
0 & 0 & 0 & 0 & 0 & 1 & 0 & 1 & 0 \\
0 & 0 & 0 & 0 & 0 & 0 & 1 & 0 & 1 \\
0 & 0 & 0 & 0 & 0 & 0 & 0 & 1 & 0
\end{array}\right]\left[\begin{array}{c}
z_{1} \\
z_{2} \\
z_{3} \\
z_{4} \\
z_{5} \\
z_{6} \\
z_{7} \\
z_{8} \\
z_{9}
\end{array}\right]=\lambda\left[\begin{array}{l}
z_{1} \\
z_{2} \\
z_{3} \\
z_{4} \\
z_{5} \\
z_{6} \\
z_{7} \\
z_{8} \\
z_{9}
\end{array}\right]
$$




$$
\left\{\begin{aligned}
z_{2}+z_{3}+z_{4} & =\lambda z_{1} \\
z_{1}+z_{5} & =\lambda z_{2} \\
z_{1} & =\lambda z_{3} \\
z_{1}+z_{6} & =\lambda z_{4} \\
z_{2} & =\lambda z_{5} \\
z_{4}+z_{7} & =\lambda z_{6} \\
z_{6}+z_{8} & =\lambda z_{7} \\
z_{7}+z_{9} & =\lambda z_{8} \\
z_{8} & =\lambda z_{9}
\end{aligned}\right.
$$

We have $z_{8}=\lambda z_{9}, z_{7}=\left(\lambda^{2}-1\right) z_{9}, z_{6}=\left(\lambda^{3}-2 \lambda\right) z_{9}$,

$z_{4}=\left(\lambda^{4}--3 \lambda^{2}+1\right) z_{9}, z_{1}=\left(\lambda^{5}-4 \lambda^{3}+3 \lambda\right) z_{9}$,

$z_{3}=\left(\lambda^{4}-4 \lambda^{2}+3\right) z_{9}, z_{2}=\left(\lambda^{6}-6 \lambda^{4}+10 \lambda^{2}-4\right) z_{9}$,

$\left.z_{5}=9 \lambda^{7}-7 \lambda^{5}=14 \lambda^{3}-7 \lambda\right) z_{9}$.

Let $z_{9}=1$. From $z_{2}=\lambda z_{5}$ we obtain

$\lambda^{6}-6 \lambda^{4}+10 \lambda^{2}-4=\lambda^{8}-7 \lambda^{6}+14 \lambda^{4}-7 \lambda^{2}$, i.e., $\lambda^{8}-8 \lambda^{6}+20 \lambda^{4}-17 \lambda^{2}+4=$ 0 . Obviously, $2^{8}+8 \cdot 2^{6}+20 \cdot 2^{4}-17 \cdot 2^{2}+4=4\left(2^{6}-2^{7}+20 \cdot 4-16\right)=$ $4(64-128+80-16)=0$, i.e., 2 is a root. Then $\vec{z}=(6,4,3,5,2,4,3,2,1)^{T}$.

Case $\tilde{A}_{n},(n \geq 2)$.

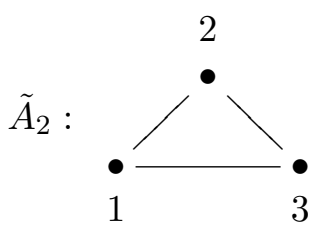

The adjacency matrix

$$
\left[\tilde{A}_{2}\right]=\left[\begin{array}{lll}
0 & 1 & 1 \\
1 & 0 & 1 \\
1 & 1 & 0
\end{array}\right]
$$

and

$$
\begin{aligned}
& {\left[\tilde{A}_{2}\right]\left(\begin{array}{l}
1 \\
1 \\
1
\end{array}\right)=2\left(\begin{array}{l}
1 \\
1 \\
1
\end{array}\right) \text {. Therefore, } r_{\tilde{A}_{2}}=2 .} \\
& \text { For } \tilde{A}_{3}:
\end{aligned}
$$

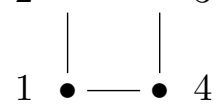


the adjacency matrix is $\left[\tilde{A}_{3}\right]=\left[\begin{array}{llll}0 & 1 & 0 & 1 \\ 1 & 0 & 1 & 0 \\ 0 & 1 & 0 & 1 \\ 1 & 0 & 1 & 0\end{array}\right]$ and $\left[\tilde{A}_{3}\right]\left(\begin{array}{l}1 \\ 1 \\ 1 \\ 1\end{array}\right)=2\left(\begin{array}{l}1 \\ 1 \\ 1 \\ 1\end{array}\right)$. Therefore, $r_{\tilde{A}_{3}}=2$.

In general case, obviously, $\left[\tilde{A}_{n}\right] \vec{z}=2 \vec{z}, \vec{z}=(1, \ldots, 1)^{T}$ and $r_{\tilde{A}_{n}}=2$.

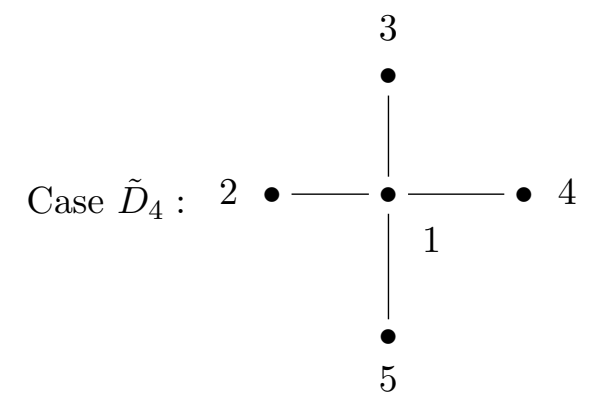

Clearly, the adjacency matrix of $\tilde{D}_{4}$ is:

$$
\begin{aligned}
& {\left[\tilde{D}_{4}\right]=\left[\begin{array}{lllll}
0 & 1 & 1 & 1 & 1 \\
1 & 0 & 0 & 0 & 0 \\
1 & 0 & 0 & 0 & 0 \\
1 & 0 & 0 & 0 & 0 \\
1 & 0 & 0 & 0 & 0
\end{array}\right] \text { and } } \\
& {\left[\begin{array}{lllll}
0 & 1 & 1 & 1 & 1 \\
1 & 0 & 0 & 0 & 0 \\
1 & 0 & 0 & 0 & 0 \\
1 & 0 & 0 & 0 & 0 \\
1 & 0 & 0 & 0 & 0
\end{array}\right]\left(\begin{array}{l}
z_{1} \\
z_{2} \\
z_{3} \\
z_{4} \\
z_{5}
\end{array}\right)=\lambda\left(\begin{array}{l}
z_{1} \\
z_{2} \\
z_{3} \\
z_{4} \\
z_{5}
\end{array}\right) . }
\end{aligned}
$$

Therefore,

$$
\begin{aligned}
& z_{2}+z_{3}+z_{4}+z_{5}=\lambda z_{1} ; \\
& z_{1}=\lambda z_{2} ; \\
& z_{1}=\lambda z_{3} ; \\
& z_{1}=\lambda z_{4} ; \\
& z_{1}=\lambda z_{5} .
\end{aligned}
$$

If $\lambda \leq 0$, then $\vec{z}$ is a non-positive eigenvector. So, $\lambda>0$ and $z_{2}=z_{3}=$ $z_{4}=z_{5}$. Let $z_{5}=1$. We obtain $z_{1}=\lambda$ and $\lambda^{2}=4$. Thus, $\lambda=2$ and $\vec{z}=(2,1,1,1,1)$. We have $r_{\tilde{D}_{4}}=2$.

For $\tilde{D}_{5}$ : 


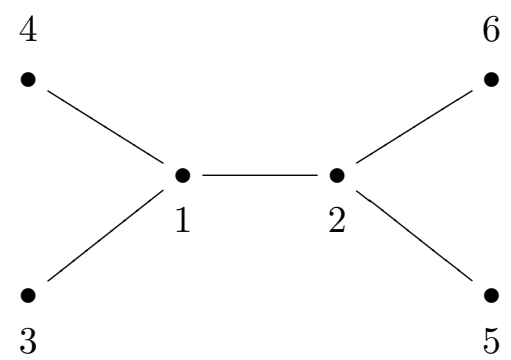

and $\left[\tilde{D}_{5}\right] \vec{z}=\lambda \vec{z}$, where $\vec{z}=\left(z_{1}, z_{2}, z_{3}, z_{4}, z_{5}, z_{6}\right)^{T}$. We have $z_{5}=z_{6}$ and $z_{3}=z_{4} \cdot z_{1}=\lambda z_{3}, z_{2}=\left(\lambda^{2}-2\right) z_{3}, z_{5}=\frac{\lambda^{3}-3 \lambda}{2} z_{3}$.

Let $z_{3}=1$. Then $\vec{z}=\left(\lambda, \lambda^{2}-2,1,1, \frac{\lambda^{3}-3 \lambda}{2}, \frac{\lambda^{3}-3 \lambda}{2}\right)^{T}$. From $z_{2}=\lambda z_{5}$ we obtain: $\lambda^{2}-2=\frac{\lambda^{4}-3 \lambda^{2}}{2}$ and $\lambda^{4}-5 \lambda^{2}+4=0.2^{4}-5 \cdot 4+4=0$. So, 2 is a root and

$\vec{z}=(2,2,1,1,1,1)^{T}$. Therefore, $r_{\tilde{D}_{5}}=2$.

Consider $\tilde{D}_{8}$ :

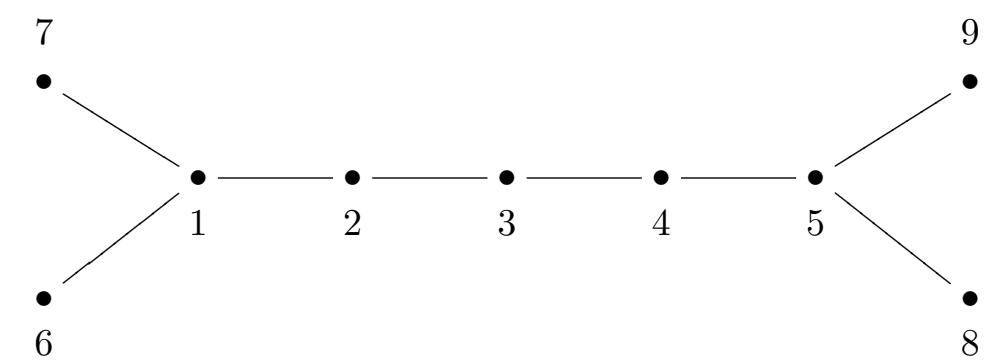

We have

$\left[\begin{array}{lllllllll}0 & 1 & 0 & 0 & 0 & 1 & 1 & 0 & 0 \\ 1 & 0 & 1 & 0 & 0 & 0 & 0 & 0 & 0 \\ 0 & 1 & 0 & 1 & 0 & 0 & 0 & 0 & 0 \\ 0 & 0 & 1 & 0 & 1 & 0 & 0 & 0 & 0 \\ 0 & 0 & 0 & 1 & 0 & 0 & 0 & 1 & 1 \\ 1 & 0 & 0 & 0 & 0 & 0 & 0 & 0 & 0 \\ 1 & 0 & 0 & 0 & 0 & 0 & 0 & 0 & 0 \\ 0 & 0 & 0 & 0 & 1 & 0 & 0 & 0 & 0 \\ 0 & 0 & 0 & 0 & 1 & 0 & 0 & 0 & 0\end{array}\right]\left[\begin{array}{l}2 \\ 2 \\ 2 \\ 2 \\ 2 \\ 1 \\ 1 \\ 1 \\ 1\end{array}\right]=2\left[\begin{array}{l}2 \\ 2 \\ 2 \\ 2 \\ 2 \\ 1 \\ 1 \\ 1 \\ 1\end{array}\right]$.

Therefore, $r_{\tilde{D}_{8}}=2$.

Consider the general case $\tilde{D}_{n}$ : 


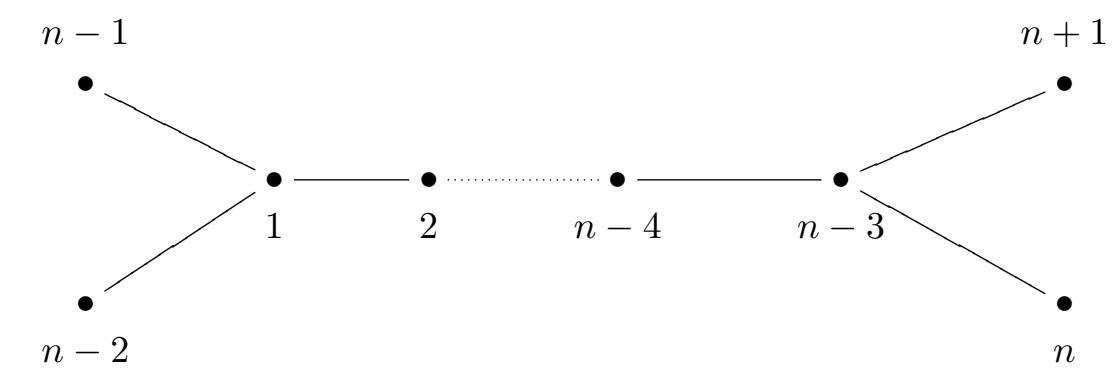

$\left.\begin{array}{c|cccccccccc}\hline 1 & 0 & 1 & 0 & 0 & \ldots & 0 & 1 & 1 & 0 & 0 \\ 2 & 1 & 0 & 1 & 0 & \ldots & 0 & 0 & 0 & 0 & 0 \\ 3 & 0 & 1 & 0 & 1 & \ldots & 0 & 0 & 0 & 0 & 0 \\ 4 & 0 & 0 & 1 & 0 & \ldots & 0 & 0 & 0 & 0 & 0 \\ \vdots & \vdots & \vdots & \vdots & \vdots & \vdots & \vdots & \vdots & \vdots & \vdots & \vdots \\ \mathrm{n}-3 & 0 & 0 & 0 & 0 & \ldots & 0 & 0 & 0 & 1 & 1 \\ \mathrm{n}-2 & 1 & 0 & 0 & 0 & \ldots & 0 & 0 & 0 & 0 & 0 \\ \mathrm{n}-1 & 1 & 0 & 0 & 0 & \ldots & 0 & 0 & 0 & 0 & 0 \\ \mathrm{n} & 0 & 0 & 0 & 0 & \ldots & 1 & 0 & 0 & 0 & 0 \\ \mathrm{n}+1 & 0 & 0 & 0 & 0 & \ldots & 1 & 0 & 0 & 0 & 0 \\ \hline & 1 & 2 & 3 & 4 & \ldots & \mathrm{n}-3 & \mathrm{n}-2 & \mathrm{n}-1 & \mathrm{n} & \mathrm{n}+1\end{array}\right]\left[\begin{array}{c}2 \\ 2 \\ 2 \\ 2 \\ \vdots \\ 2 \\ 1 \\ 1 \\ 1 \\ 1\end{array}\right]=2\left[\begin{array}{c}2 \\ 2 \\ 2 \\ 2 \\ \vdots \\ 2 \\ 1 \\ 1 \\ 1 \\ 1\end{array}\right]$

Thus, $r_{\tilde{D}_{n}}=2$.

\section{Corollary 4.1.}

(a) For each extended Dynkin diagram $G \in\left\{\tilde{A}_{n}, \tilde{D}_{n}, \tilde{E}_{6}, \tilde{E}_{7}, \tilde{E}_{8}\right\} r_{G}=2$.

(b) For each Dynkin diagram $G \in\left\{A_{n}, D_{n}, E_{6}, E_{7}, E_{8}\right\}$ we have $r_{G}<2$.

Proof. (a) For any extended Dynkin diagram $G$ we already gave a positive eigenvector with eigenvalue 2 . Therefore, $r_{G}=2$.

(b) We have the following inclusions: $A_{n} \subset \tilde{A}_{n}, D_{n} \subset \tilde{D}_{n}, E_{6} \subset \tilde{E}_{6}, E_{7} \subset$ $\tilde{E}_{7}, E_{8} \subset \tilde{E}_{8}$. By Theorem $2.1 r_{G}<2$ for any $G \in\left\{A_{n}, D_{n}, E_{6}, E_{7}, E_{8}\right\}$.

Proof of Smith's theorem. Corollary 4.1 gives the "if" part of Smith's theorem.

Conversely, let $G$ be a connected finite simple graph with $r_{G} \leq 2$. If $G$ is not a tree, then $G$ must be the extended Dynkin diagram $\tilde{A}_{n}$. So, $G$ is a tree. It is easy to see $G$ must be a tree of the form $T_{p, q, r}$ (see [31, Exercise 4.3]). Using Theorem 2.1 we obtain that $T_{p, q, r}$ is either one of simply laced Dynkin diagrams or one of simply laced extended Dynkin diagrams. 


\section{Some examples}

Let $E_{6}$ be given in the form:

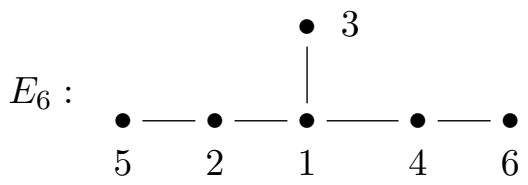

Then

$$
\begin{aligned}
& {\left[\begin{array}{cccccc}
0 & 1 & 1 & 1 & 0 & 0 \\
1 & 0 & 0 & 0 & 1 & 0 \\
1 & 0 & 0 & 0 & 0 & 0 \\
1 & 0 & 0 & 0 & 0 & 1 \\
0 & 1 & 0 & 0 & 0 & 0 \\
0 & 0 & 0 & 1 & 0 & 0
\end{array}\right]\left(\begin{array}{l}
z_{1} \\
z_{2} \\
z_{3} \\
z_{4} \\
z_{5} \\
z_{6}
\end{array}\right)=\lambda\left(\begin{array}{l}
z_{1} \\
z_{2} \\
z_{3} \\
z_{4} \\
z_{5} \\
z_{6}
\end{array}\right) } \\
& z_{2}+z_{3}+z_{4}=\lambda z_{1} \\
& z_{1}+z_{5}=\lambda z_{2} \\
& z_{1}=\lambda z_{3} \\
& z_{1}+z_{6}=\lambda z_{4} \\
& z_{2}=\lambda z_{5} \\
& z_{4}=\lambda z_{6}
\end{aligned}
$$

Let $z_{6}=1$. Then $z_{4}=\lambda, z_{)} 1=\lambda^{2}-1$ and $z_{3}=\frac{\lambda^{2}-1}{\lambda}$. Obviously, $z_{2}=\frac{\lambda_{4}-3 \lambda^{2}+1}{\lambda}$. Therefore, $z_{5}=\frac{\lambda^{4}-3 \lambda^{2}+1}{\lambda^{2}}$. On the other hand, $z_{5}=$ $\lambda z_{2}-z_{1}=\lambda^{4}-4 \lambda^{2}+2$. Consequently, $\frac{\lambda^{4}-3 \lambda^{2}+1}{\lambda^{2}}=\lambda^{4}-4 \lambda^{2}+2$. We obtain that $\lambda^{6}-5 \lambda^{4}+5 \lambda^{2}-1=0$.

Let $E_{7}$ be given as follows:

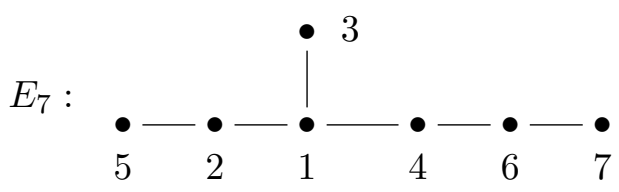

Then

$$
\left[\begin{array}{lllllll}
0 & 1 & 1 & 1 & 0 & 0 & 0 \\
1 & 0 & 0 & 0 & 1 & 0 & 0 \\
1 & 0 & 0 & 0 & 0 & 0 & 0 \\
1 & 0 & 0 & 0 & 0 & 1 & 0 \\
0 & 1 & 0 & 0 & 0 & 0 & 0 \\
0 & 0 & 0 & 1 & 0 & 0 & 1 \\
0 & 0 & 0 & 0 & 0 & 1 & 0
\end{array}\right]\left(\begin{array}{l}
z_{1} \\
z_{2} \\
z_{3} \\
z_{4} \\
z_{5} \\
z_{6} \\
z_{7}
\end{array}\right)=\lambda\left(\begin{array}{l}
z_{1} \\
z_{2} \\
z_{3} \\
z_{4} \\
z_{5} \\
z_{6} \\
z_{7}
\end{array}\right)
$$




$$
\begin{aligned}
z_{2}+z_{3}+z_{4} & =\lambda z_{1} \\
z_{1}+z_{5} & =\lambda z_{2} \\
z_{1} & =\lambda z_{3} \\
z_{1}+z_{6} & =\lambda z_{4} \\
z_{2} & =\lambda z_{5} \\
z_{4}+z_{7} & =\lambda z_{6} \\
z_{6} & =\lambda z_{7}
\end{aligned}
$$

Let $z_{7}=1$ and $z_{6}=\lambda$. Then $z_{4}=\lambda z_{6}-z_{7}$. We obtain $z_{4}=\lambda^{2}-1$. Therefore, $z_{1}=\lambda^{3}-2 \lambda$. Obviously, $z_{3}=\lambda^{2}-2$. We have $z_{2}=\lambda z_{1}-$ $z_{3}-z_{4}$ and $z_{2}=\lambda^{4}-4 \lambda^{2}+3$. From the equality $z_{2}=\lambda z_{5}$ it follows that $z_{5}=\frac{\lambda^{4}-4 \lambda^{2}+3}{\lambda}$. On the other hand, $z_{5}=\lambda z_{2}-z_{1}=\lambda^{5}-5 \lambda^{3}+5 \lambda$. So, $\frac{\lambda^{4}-4 \lambda^{2}+3}{\lambda}=\lambda^{5}-5 \lambda^{3}+5 \lambda$ and $\lambda^{6}-6 \lambda^{4}+9 \lambda^{2}-3=0$.

Let $E_{8}$ be given in the following form:

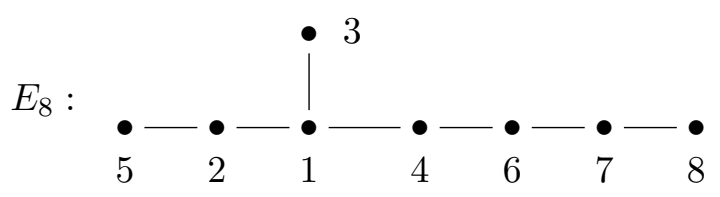

Then

$$
\left[\begin{array}{llllllll}
0 & 1 & 1 & 1 & 0 & 0 & 0 & 0 \\
1 & 0 & 0 & 0 & 1 & 0 & 0 & 0 \\
1 & 0 & 0 & 0 & 0 & 0 & 0 & 0 \\
1 & 0 & 0 & 0 & 0 & 1 & 0 & 0 \\
0 & 1 & 0 & 0 & 0 & 0 & 0 & 0 \\
0 & 0 & 0 & 1 & 0 & 0 & 1 & 0 \\
0 & 0 & 0 & 0 & 0 & 1 & 0 & 1 \\
0 & 0 & 0 & 0 & 0 & 0 & 1 & 0
\end{array}\right]\left(\begin{array}{c}
z_{1} \\
z_{2} \\
z_{3} \\
z_{4} \\
z_{5} \\
z_{6} \\
z_{7} \\
z_{8}
\end{array}\right)=\lambda\left(\begin{array}{l}
z_{1} \\
z_{2} \\
z_{3} \\
z_{4} \\
z_{5} \\
z_{6} \\
z_{7} \\
z_{8}
\end{array}\right)
$$

We obtain the following system of linear equations

$$
\begin{aligned}
z_{2}+z_{3}+z_{4} & =\lambda z_{1} \\
z_{1}+z_{5} & =\lambda z_{2} \\
z_{1} & =\lambda z_{3} \\
z_{1}+z_{6} & =\lambda z_{4} \\
z_{2} & =\lambda z_{5} \\
z_{4}+z_{7} & =\lambda z_{6} \\
z_{6}+z_{8} & =\lambda z_{7} \\
z_{7} & =\lambda z_{8}
\end{aligned}
$$

Let $z_{8}=1$. Then $z_{7}=\lambda$ and $z_{6}=\lambda^{2}-1$. Obviously, we have: $z_{4}=$ $\lambda^{3}-2 \lambda, z_{1}=\lambda^{4}-3 \lambda^{2}+1, z_{3}=\frac{\lambda^{4}-3 \lambda^{2}+1}{\lambda}, z_{2}=\frac{\lambda^{6}-5 \lambda^{4}+6 \lambda^{2}-1}{\lambda}$ and $z_{5}=$ $\frac{\lambda^{6}-5 \lambda^{4}+6 \lambda^{2}-1}{\lambda}$. 
Hence, $z_{5}=\lambda z_{2}-z_{1}=\lambda^{6}-6 \lambda^{4}+9 \lambda^{2}-2$. Consequently, $\lambda^{6}-6 \lambda^{4}+$ $9 \lambda^{2}-2=\frac{\lambda^{6}-5 \lambda^{4}+6 \lambda^{2}-1}{\lambda^{2}}$ and $\lambda^{8}-7 \lambda^{6}+14 \lambda^{4}-8 \lambda^{2}+1=0$

In conclusion we consider the following simple graph $G_{5}$ :

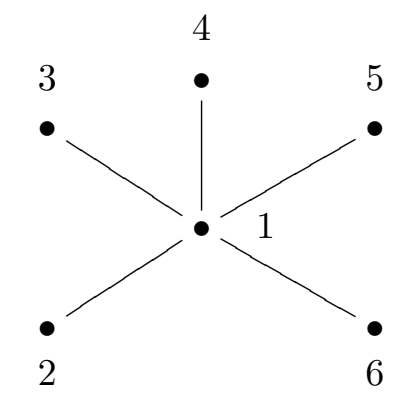

with the adjacency matrix $\left[G_{5}\right]$ :

$$
\left(\begin{array}{llllll}
0 & 1 & 1 & 1 & 1 & 1 \\
1 & 0 & 0 & 0 & 0 & 0 \\
1 & 0 & 0 & 0 & 0 & 0 \\
1 & 0 & 0 & 0 & 0 & 0 \\
1 & 0 & 0 & 0 & 0 & 0 \\
1 & 0 & 0 & 0 & 0 & 0
\end{array}\right)
$$

We have

$$
\left[G_{5}\right] \vec{z}=\lambda \vec{z},
$$

where $\vec{z}=\left(z_{1}, z_{2}, z_{3}, z_{4}, z_{5}, z_{6}\right)^{T}$. From 1 we obtain

$$
\begin{aligned}
z_{2}+z_{3}+z_{4}+z_{5}+z_{6} & =\lambda z_{1} \\
z_{1} & =\lambda z_{2} \\
z_{1} & =\lambda z_{3} \\
z_{1} & =\lambda z_{4} \\
z_{1} & =\lambda z_{5} \\
z_{1} & =\lambda z_{6}
\end{aligned}
$$

Consequently, $5 z_{1}=\lambda\left(z_{2}+z_{3}+z_{4}+z_{5}+z_{6}\right)=\lambda^{2} z_{1}$. Since, $z_{1} \neq 0$, we obtain $\lambda=\sqrt{5}$ and $\vec{z}=(\sqrt{5}, 1,1,1,1,1)$ and $r_{G_{5}}=\sqrt{5}>2$.

\section{Acknowledgements}

The first and the third authors were partially supported by Fapesp and $\mathrm{CNPq}$ (Brazil). The second and the last authors were supported by 
FAPESP (Brazil), and they thank the Department of Mathematics of the University of São Paulo for its warm hospitality during their visit in 2010.

\section{References}

[1] V. Arnold, The A-D-E-classifications. Mathematical developments arising from Hilbert problems (ed. F.E. Browder). Proceedings Symposia Pure Math. 28, Providence (1976), 46.

[2] M. Auslander, I. Reiten, S.O. Smalø, Representation theory of Artin algebras. Cambridge Studies in Advanced Mathematics, 36. Cambridge University Press, Cambridge, 1995.

[3] M. Auslander, I. Reiten, McKay quivers and extended Dynkin diagrams. Trans. Amer. Math. Soc. 293:1 (1986), 293-301.

[4] S. Berman, R. Moody, M. Wonenburger, Cartan matrices with null roots and finite Cartan matrices. Indian Math. J. 21 (1972), 1091-1099.

[5] I.N. Bernstein, I.M. Gelfand and V.A. Ponomarev, Coxeter functors and Gabriel's theorem. Usp. Mat. Nauk. 28 (1973), 19-33, Transl. Russ. Math. Surv. 28 (1973), $17-32$.

[6] N. Bourbaki, Elements de mathematique, Groupes et algebras de Lie, Chap. 4-6, Hermann, Paris, 1968.

[7] P.J. Cameron, J.-M. Goethals, J.J. Seidel, E.E. Shult, Line graphs, root systems, and elliptic geometry. J. Algebra 43:1 (1976), 305-327.

[8] H.S.M. Coxeter, Regular polytopes. Third edition. Dover Publications, Inc., New York, 1973.

[9] D.M Cvetković, M.S. Doob, H. Sachs, Spectra of graphs. Theory and application. Pure and Applied Mathematics, 87. Academic Press Inc., New York-London, 1980.

[10] D.M. Cvetković, M.S. Doob, I. Gutman, A. Torgasev, Recent results in the theory of graph spectra. Annals of Discrete Mathematics, 36. North-Holland Publishing Co., Amsterdam, 1988.

[11] D.M. Cvetković, I. Gutman, On spectral structure of graphs having the maximal eigenvalue not greater than two. Publ. Inst. Math. Beograd (N.S.) 18:32 (1975), 39-45.

[12] D.M. Cvetković, P. Rowlinson, S. Simić, Spectral Generalizations of Line Graphs. On graphs with least eigenvalue - 2. Cambridge Univ. Press, 2004.

[13] V. Dlab, C.M. Ringel, Indecomposable representations of graphs and algebras. Mem. Amer. Math. Soc. 6 (1976), no. 173.

[14] V. Dlab, C.M. Ringel, On algebras of finite representation type. J. Algebra 33 (1975), 306-394.

[15] V. Dlab, C.M. Ringel, Representations of graphs and algebras. Carleton Mathematical Lecture Notes, 8. Department of Mathematics, Carleton University, Ottawa, Ont., 1974.

[16] P. Donovan and M.-R. Freislish, The representation theory of finite graphs and associated algebras. Carleton Math. Lecture Notes 5 (1973).

[17] Yu.A. Drozd, Tame and wild matrix problems. (Russian) Matrix problems (Russian), 104-114. Akad. Nauk Ukrain. SSR Inst. Mat., Kiev, 1977.

[18] Yu.A. Drozd, Tame and wild matrix problems. (Russian) Representations and quadratic forms (Russian), 39-74, 154, Akad. Nauk Ukrain. SSR, Inst. Mat., Kiev, 1979. 
[19] Yu.A. Drozd, Tame and wild matrix problems. Representation theory, II (Proc. Second Internat. Conf., Carleton Univ., Ottawa, Ont., 1979), 242-258, Lecture Notes in Math., 832, Springer, Berlin-New York, 1980.

[20] E.B. Dynkin, Classification of the simple Lie groups. (Russian) Rec. Math., Mat. Sbornik (N. S.) 18:60 (1946), 347-352.

[21] E.A. Fernandez, Graphs and adjacency matrices. (Spanish) Proceedings of the 8th "Dr. Antonio A. R. Monteiro" Congress (Spanish), 151-179, Actas Congr. "Dr. Antonio A. R. Monteiro", Univ. Nac. del Sur, Baha Blanca, 2006.

[22] P. Gabriel, Unzerlegbare Dartellungen I, Manuscr. Math, 6 (1972), 71-103.

[23] P. Gabriel, Indecomposable representations. II. Symposia Mathematica, Vol. XI (Convegno di Algebra Commutativa, INDAM, Rome, 1971), Academic Press, London, 1973, 81-104.

[24] P. Gabriel and A.V. Roiter, Representation of Finite-Dimensional Algebras. Springer-Verlag, Berlin, 1997.

[25] F.R. Gantmacher, The theory of matrices, 2, Chelsea, 1960.

[26] I.M. Gelfand Lectures on linear algebra. (Russian) Izdat. "Nauka", Moscow, 1971.

[27] F.M. Goodman, P. de la Harpe, V.F.R. Jones, Coxeter Graphs and Towers of Algebras. Springer-Verlag, New York, Berlin, Heidelberg, London, Paris, Tokyo, 1989.

[28] D. Happel, U. Preiser, C.M. Ringel, Vinberg's characterization of Dynkin diagrams using subadditive functions with application to DTr-periodic modules. Representation theory, II (Proc. Second Internat. Conf., Carleton Univ., Ottawa, Ont., 1979), 280-294, Lecture Notes in Math., 832, Springer, Berlin, 1980.

[29] M. Hazewinkel, N. Gubareni and V.V. Kirichenko, Algebras, rings and modules. Vol. 2. Mathematics and Its Applications (Springer), 586. Springer, Dordrecht, 2007.

[30] Hazewinkel, W. Hesselink, D. Sierma and F.D. Veldkamp, The ubiquity of Coxeter-Dynkin diagrams (an introduction to the A-D-E problem), Nieuw Arch. Wiskunde, III. Ser. 25 (1977), 257-307.

[31] V.G. Kac, Infinite-dimensional Lie algebras. Third edition. Cambridge University Press, Cambridge, 1990.

[32] L. Kronecker, Zwein Sätze über Gleichungen mit ganzzahligen Coefficienten. J. Reine Angew. Math. 53 (1857), 173-175.

[33] P.W.H. Lemmens, J.J. Seidel, Equiangular lines. J. Algebra 24 (1973), 494-512.

[34] L. Lovász, J. Pelikán, On the eigenvalues of trees. Collection of articles dedicated to the memory of Alfred Renyi, II. Period. Math. Hungar. 3 (1973), 175-182.

[35] I.G. Macdonald, Kac-Moody algebras. Lie algebras and related topics (Windsor, Ont., 1984), CMS Conf. Proc., 5, Amer. Math. Soc., Providence, RI, 1986, 69109.

[36] R.V. Moody, A. Pianzola, Lie algebras with triangular decompositions. Canadian Mathematical Society Series of Monographs and Advanced Texts. A WileyInterscience Publication. John Wiley \& Sons, Inc., New York, 1995.

[37] Yu.P. Moskaleva and Yu.S. Samoilenko, Introduction in spectral theory of graphs. Center textbooks. (Russian) Kiev, 2007.

[38] L.A. Nazarova, A.V. Roiter, The norm of a relation, separation functions, and representations of marked quivers. (Russian) Ukran. Mat. Zh. 54:6 (2002), 808840; translation in Ukrainian Math. J. 54:6 (2002), 990-1018.

[39] L.A. Nazarova, Representations of quivers of infinite type. Izv. Akad. Nauk SSSR 37:4 (1973), 752-791.

[40] J.A. de la Peña and M. Takane, Spectral properties of Coxeter transformations and applications. Archiv der Math. 55 (1990), 157-174. 
104M. A. Dokuchaev, N. M. Gubareni, V. M. Futorny, M.A. Khibina, and V. V. Kirichenko

[41] J.H. Smith, Some properties of the spectrum of a graph, in: Combinatorial Structures and their Applications (Eds. R. Guy, H. Hanani, N. Sauer, J. Schönheim), Gordon and Breach (New York), 1970, 403-406.

[42] E.B. Vinberg, Discrete linear groups generated by reflections. Izv. Akad. Nauk SSSR. Ser. Mat. 35 (1971), Engl. translation: Math. USSR Izv. 5 (1971), 10831119 .

São Paulo J.Math.Sci. 7, 1 (2013), 83-104 